\title{
Photolithography technology in electronic fabrication
}

\author{
Xiao Ming $\mathrm{Hu}^{1}$ \\ ${ }^{1}$ Dept. electronic \& electrical engineering, Wuhan railway vocational college of technology, Wuhan, \\ Chain
}

Email: huxiaolili@tom.com, Wuhan jiangxia district hidden dragon road, no.1, 430205

Keywords: photolithography, photomask, EUV(12nm)

\begin{abstract}
The paper introduce the photolithography technology. First, the writer explain the process of photolithography. a modern wafer (form IC) will go through a photolithography cycle up to 50 times, some 100 times more. then, the article illustrate photoresist, photoresists are classified two groups :positive resist and negative resist. another important technology is photomask, it is the mass production of IC device,worldwide photomask market was estimated as 3.2 billion in 2012.at the last ,the paper introduce the photolithography machine(tools). The newest feature size of photolithography machine will bring us to 12nm time, maybe 2016.
\end{abstract}

\section{Photolithography technology}

Photolithography, also termed optical lithography or UV lithography, is a process used in microfabrication to pattern parts of a thin film or the bulk of a substrate. It uses light to transfer a geometric pattern from a photomask to a light-sensitive chemical "photoresist", or simply "resist," on the substrate. A series of chemical treatments then either engraves the exposure pattern into, or enables deposition of a new material in the desired pattern upon, the material underneath the photo resist. For example, in complex integrated circuits, a modern CMOS wafer will go through the photolithographic cycle up to 50 times.

Simplified illustration of dry etching using positive photoresist during a photolithography process in semiconductor microfabrication (not to scale).

A single iteration of photolithography combines several steps in sequence. Modern cleanrooms use automated, robotic wafer track systems to coordinate the process. The procedure described here omits some advanced treatments, such as thinning agents or edge-bead removal.[1]

\section{Preparation and Cleaning}

The wafer is initially heated to a temperature sufficient to drive off any moisture that may be present on the wafer surface, $150^{\circ} \mathrm{C}$ for ten minutes is sufficient. Wafers that have been in storage must be chemically cleaned to remove contamination. A liquid or gaseous "adhesion promoter", such as Bis(trimethylsilyl)amine (HMDS), is applied to promote adhesion of the photoresist to the wafer. The surface layer of silicon dioxide on the wafer reacts with HMDS to form tri-methylated silicon-dioxide, a highly water repellent layer not unlike the layer of wax on a car's paint. This water repellent layer prevents the aqueous developer from penetrating between the photoresist layer and the wafer's surface, thus preventing so-called lifting of small photoresist structures in the (developing) pattern. In order to ensure the development of the image, it is best covered and placed over a hot plate and let it dry while stabilizing the temperature at $120^{\circ} \mathrm{C} .^{[3]}$

If organic or inorganic contaminations are present on the wafer surface, they are usually removed by wet chemical treatment, e.g. theRCA clean procedure based on solutions containing hydrogen peroxide. Other solutions can also be used to clean. ${ }^{[2]}$

The wafer is covered with photoresist by spin coating. A viscous, liquid solution of photoresist is dispensed onto the wafer, and the wafer is spun rapidly to produce a uniformly thick layer. The spin coating typically runs at 1200 to $4800 \mathrm{rpm}$ for 30 to 60 seconds, and produces a layer between 0.5 and 2.5 micrometres thick. The spin coating process results in a uniform thin layer, usually with uniformity of within 5 to 10 nanometres. This uniformity can be explained by detailed 
fluid-mechanical modelling, which shows that the resist moves much faster at the top of the layer than at the bottom, where viscous forces bind the resist to the wafer surface. Thus, the top layer of resist is quickly ejected from the wafer's edge while the bottom layer still creeps slowly radially along the wafer. In this way, any 'bump' or 'ridge' of resist is removed, leaving a very flat layer. Final thickness is also determined by the evaporation of liquid solvents from the resist. For very small, dense features $(<125$ or so $\mathrm{nm}$ ), lower resist thicknesses $(<0.5$ micrometres) are needed to overcome collapse effects at high aspect ratios; typical aspect ratios are $<4: 1$.

The photo resist-coated wafer is then prebaked to drive off excess photoresist solvent, typically at 90 to $100^{\circ} \mathrm{C}$ for 30 to 60 seconds on a hotplate.

\section{Exposure and Etching}

After prebaking, the photoresist is exposed to a pattern of intense light. The exposure to light causes a chemical change that allows some of the photoresist to be removed by a special solution, called "developer" by analogy with photographic developer. Positive photoresist, the most common type, becomes soluble in the developer when exposed; with negative photoresist, unexposed regions are soluble in the developer.

A post-exposure bake (PEB) is performed before developing, typically to help reduce standing wave phenomena caused by the destructive and constructive interference patterns of the incident light. In deep ultraviolet lithography, chemically amplified resist (CAR) chemistry is used. This process is much more sensitive to PEB time, temperature, and delay, as most of the "exposure" reaction (creating acid, making the polymer soluble in the basic developer) actually occurs in the PEB. ${ }^{[4]}$

The develop chemistry is delivered on a spinner, much like photoresist. Developers originally often contained sodium hydroxide $(\mathrm{NaOH})$. However, sodium is considered an extremely undesirable contaminant in MOSFET fabrication because it degrades the insulating properties of gate oxides (specifically, sodium ions can migrate in and out of the gate, changing the threshold voltage of the transistor and making it harder or easier to turn the transistor on over time).

In semiconductor fabrication, dry etching techniques are generally used, as they can be made anisotropic, in order to avoid significant undercutting of the photoresist pattern. This is essential when the width of the features to be defined is similar to or less than the thickness of the material being etched (i.e. when the aspect ratio approaches unity). Wet etch processes are generally isotropic in nature, which is often indispensable for microelectromechanical systems, where suspended structures must be "released" from the underlying layer.

The development of low-defectivity anisotropic dry-etch process has enabled the ever-smaller features defined photolithographically in the resist to be transferred to the substrate material.

After a photoresist is no longer needed, it must be removed from the substrate. This usually requires a liquid "resist stripper", which chemically alters the resist so that it no longer adheres to the substrate. Alternatively, photoresist may be removed by a plasma containing oxygen, which oxidizes it. This process is called ashing, and resembles dry etching. 1-Methyl-2-pyrrolidon (NMP) solvent is another method used to remove an image. NMP is soluble with photoresist and has a high boiling point, thus when the resist has dissolved off the solution and wafer can be heated up to $80^{\circ} \mathrm{C}$ without leaving any residue. ${ }^{[5]}$

Photolithography has been defeating predictions of its demise for many years. For instance, by the early 1980s, many in the semiconductor industry had come to believe that features smaller than 1 micrometer could not be printed optically. Modern techniques using excimer laser lithography already print features with dimensions a fraction of the wavelength of light used - an amazing optical feat. New tricks such as immersion lithography, dual-tone resist and multiple patterning continue to improve the resolution of $193 \mathrm{~nm}$ lithography. Meanwhile, current research is exploring alternatives to conventional UV, such as electron beam lithography, X-ray lithography, extreme ultraviolet lithography and ion projection lithography. 


\section{Photoresist}

A photoresist is a light-sensitive material used in several industrial processes, such as photolithography and photoengraving to form a patterned coating on a surface.

The photoresist main types are: a positive tone resist and a negative tone resist.

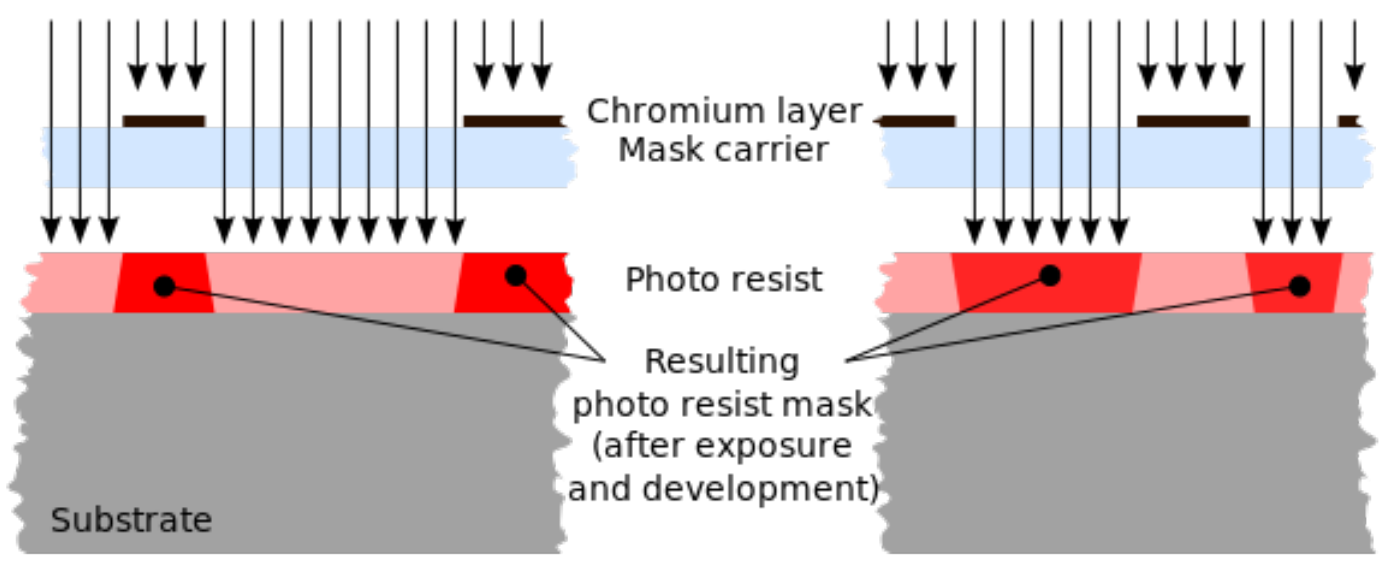

\section{Positive tone resist Negative tone resist}

Fig.1 positive and negative resist

Comparison between a positive tone resist and a negative tone resist. Photoresists are classified into two groups: positive resists and negative resists.

A positive resist is a type of photoresist in which the portion of the photoresist that is exposed to light becomes soluble to the photoresist developer. The portion of the photoresist that is unexposed remains insoluble to the photoresist developer.

A negative resist is a type of photoresist in which the portion of the photoresist that is exposed to light becomes insoluble to the photoresist developer. The unexposed portion of the photoresist is dissolved by the photoresist developer.

Table 1 Differences between tone types ${ }^{[6]}$

\begin{tabular}{|c|c|c|}
\hline Characteristic & Positive & Negative \\
\hline Adhesion to Silicon & Fair & Excellent \\
\hline Relative Cost & More Expensive & $\begin{array}{c}\text { Less } \\
\text { Expensive }\end{array}$ \\
\hline Developer Base & Aqueous & Organic \\
\hline Minimum Feature & $0.5 \mu \mathrm{m}$ and below & $2 \mu \mathrm{m}$ \\
\hline Step Coverage & Better & Lower \\
\hline Wet Chemical Resistance & Fair & Excellent \\
\hline
\end{tabular}

Note: This table is based on the MEMS fabrication industry. 


\section{Applications}

Negative photoresist.Contrary to past types, current negative photoresists tend to exhibit better adhesion to various substrates such as $\mathrm{Si}, \mathrm{GaAs}, \mathrm{InP}$ and glass, as well as metals, including $\mathrm{Au}, \mathrm{Cu}$ and $\mathrm{Al}$, compared to positive-tone photoresists. Additionally, the current generation of $\mathrm{G}, \mathrm{H}$ and I-line negative-tone photoresists exhibit higher temperature resistance over positive resists.

One very common negative photoresist is based on epoxy-based polymer. The common product name is SU-8 photoresist, and it was originally invented by IBM, but is now sold by Microchem and Gersteltec. One unique property of SU-8 is that it is very difficult to strip. As such, it is often used in applications where a permanent resist pattern (one that is not strippable, and can even be used in harsh temperature and pressure environments) is needed for a device.

DUVphotoresist.Deep ultraviolet (DUV) resists are typically polyhydroxystyrene-based polymers with a photoacid generator providing the solubility change. However, this material does not experience the diazocoupling. The combined benzene-chromophore and DNQ-novolac absorption mechanisms lead to stronger absorption by DNQ-novolac photoresists in the DUV, requiring a much larger amount of light for sufficient exposure. The strong DUV absorption results in diminished photoresist sensitivity.

Fabrication of printed circuit boards. This can be done by applying photoresist, exposing to the image, and then etching using iron chloride, cupric chloride or an alkaline ammonia etching solution to remove the copperclad substrate.

Microelectronics. This application, mainly applied to silicon wafers/silicon integrated circuits is the most developed of the technologies and the most specialized in the field.

Patterning and etching of substrates. This includes specialty photonics materials, MEMS, glass printed circuit boards, and other micropatterning tasks. Photoresist tends not to be etched by solutions with a $\mathrm{pH}$ greater than $3 .^{[7]}$

\section{Photomask}

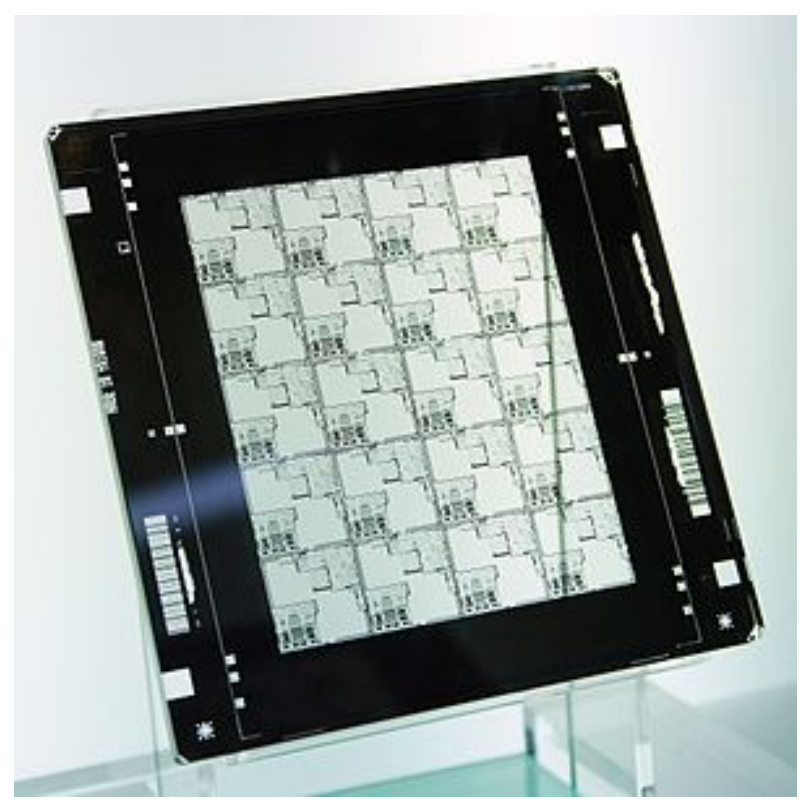

Fig.2 schematic illustration of a photomask 


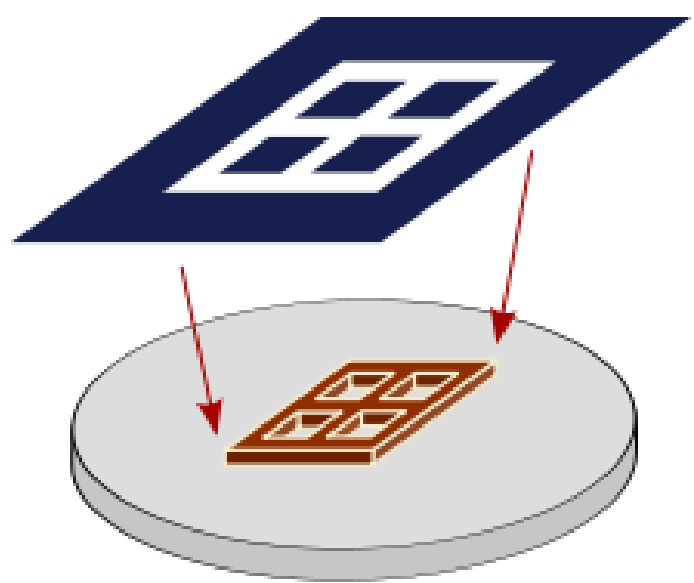

Fig.3 an integrated circuit created using that mask

A photomask is an opaque plate with holes or transparencies that allow light to shine through in a defined pattern. They are commonly used in photolithography.

Lithographic photomasks are typically transparent fused silica blanks covered with a pattern defined with a chrome metal-absorbing film. Photomasks are used at wavelengths of $365 \mathrm{~nm}, 248$ $\mathrm{nm}$, and $193 \mathrm{~nm}$. Photomasks have also been developed for other forms of radiation such as $157 \mathrm{~nm}$, $13.5 \mathrm{~nm}$ (EUV), X-ray, electrons, and ions; but these require entirely new materials for the substrate and the pattern film.

A set of photomasks, each defining a pattern layer in integrated circuit fabrication, is fed into a photolithography stepperor scanner, and individually selected for exposure. In double patterning techniques, a photomask would correspond to a subset of the layer pattern.

In photolithography for the mass production of integrated circuit devices, the more correct term is usually photoreticle or simply reticle. In the case of a photomask, there is a one-to-one correspondence between the mask pattern and the wafer pattern. This was the standard for the 1:1 mask aligners that were succeeded by steppers and scanners with reduction optics. As used in steppers and scanners, the reticle commonly contains only one layer of the chip. (However, some photolithography fabrications utilize reticles with more than one layer patterned onto the same mask). The pattern is projected and shrunk by four or five times onto the wafer surface. ${ }^{[8]}$ To achieve complete wafer coverage, the wafer is repeatedly "stepped" from position to position under the optical column until full exposure is achieved.

Features $150 \mathrm{~nm}$ or below in size generally require phase-shifting to enhance the image quality to acceptable values. This can be achieved in many ways. The two most common methods are to use an attenuated phase-shifting background film on the mask to increase the contrast of small intensity peaks, or to etch the exposed quartz so that the edge between the etched and unetched areas can be used to image nearly zero intensity. In the second case, unwanted edges would need to be trimmed out with another exposure. The former method is attenuated phase-shifting, and is often considered a weak enhancement, requiring special illumination for the most enhancement, while the latter method is known as alternating-aperture phase-shifting, and is the most popular strong enhancement technique.

As leading-edge semiconductor features shrink, photomask features that are $4 \times$ larger must inevitably shrink as well. This could pose challenges since the absorber film will need to become thinner, and hence less opaque. ${ }^{[9]}$ A recent study by IMEChas found that thinner absorbers degrade image contrast and therefore contribute to line-edge roughness, using state-of-the-art photolithography tools. One possibility is to eliminate absorbers altogether and use "chromeless" masks, relying solely on phase-shifting for imaging.

The emergence of immersion lithography has a strong impact on photomask requirements. The commonly used attenuated phase-shifting mask is more sensitive to the higher incidence angles applied in "hyper-NA" lithography, due to the longer optical path through the patterned film. 


\section{Photolithography Machine}

Photolithography machine is the bibcock of microelectronic equipment, The technical difficulty of the highest, and The biggest single equipment cost very high, The integrated density,so, The Lithography machine is the leading source of photolithography, We say ,Lithography just Transfer the pattern of circuitry from a mask onto a wafer.

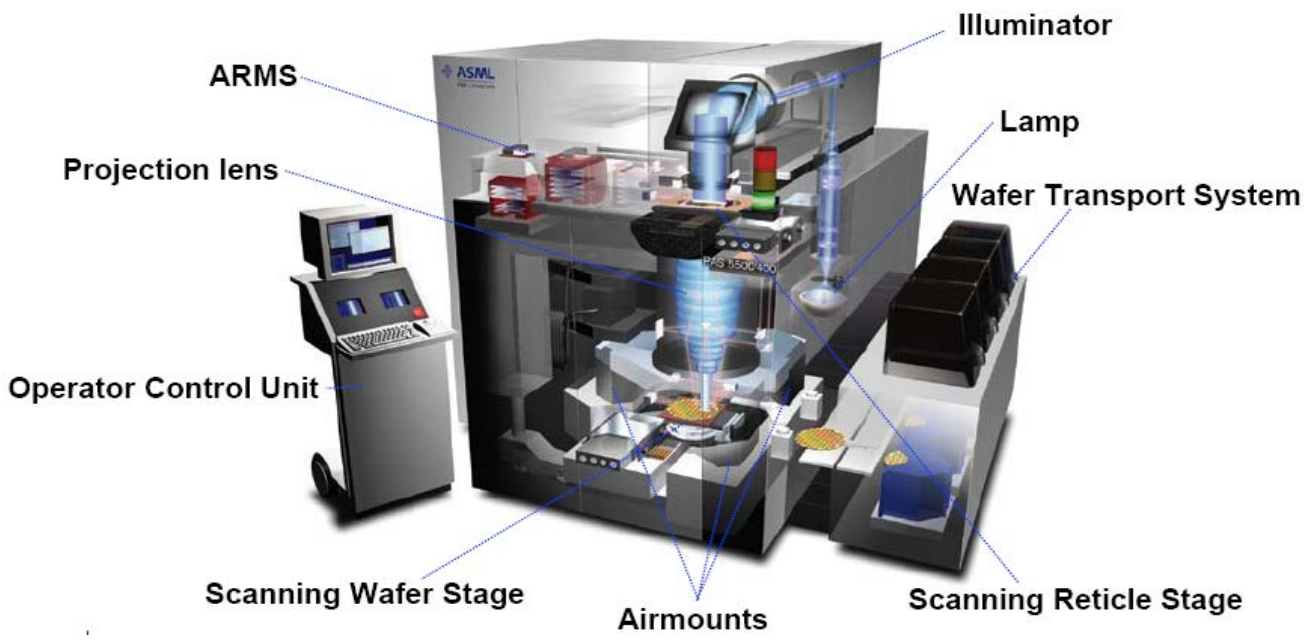

Fig.4 The overall structure of photoetching machine

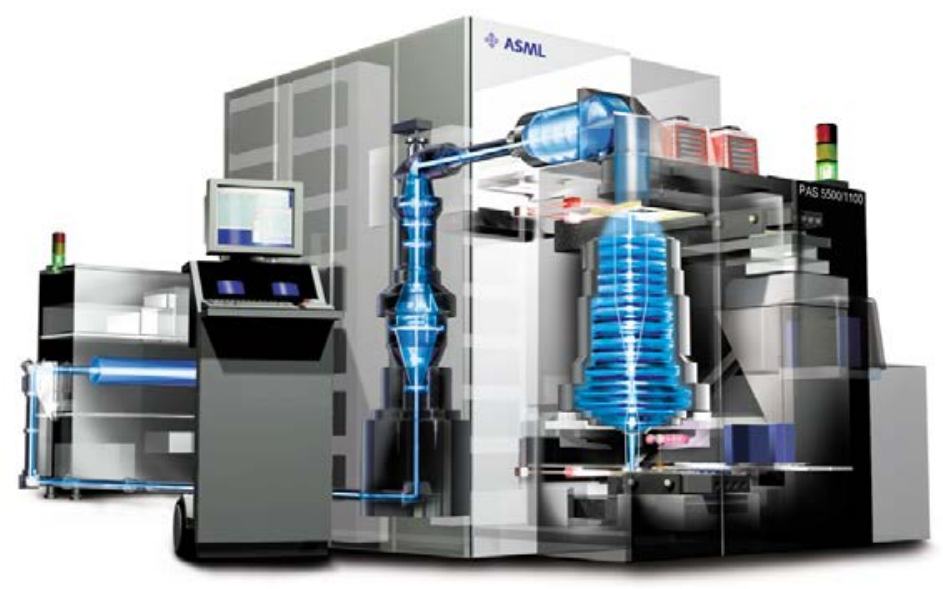

Fig.5 Step and Scan System

we know, The exposure wavelength

Mercury lamp:g-Line (453 nm)、h-Line (405 nm)、i-Line (365 nm)

Excimer laser:KrF (248 nm)、ArF (193 nm)、F2 (157 nm)

Extreme ultraviolet light source: EUV $(13 \mathrm{~nm})$

The most important light types include UV, DUV (Deep UV), and the g and I lines having wavelength of $436 \mathrm{~nm}$ and $365 \mathrm{~nm}$ respectively of a mercury-vapor lamp.

This particular parameter is closely related to the thickness of the applied photoresist, with thinner layers corresponding to shorter wavelengths, permitting a reduced aspect ratio and a reduced minimum feature size. This is important in microelectronics and especially the ITRS reduction in minimum feature size. Intel has semiconductor fabrication facilities currently operating at the 22 nanometer node. 
Table 2 The development of lithography tools

\begin{tabular}{|c|c|c|c|}
\hline \multicolumn{4}{|c|}{ The development of lithography machine } \\
\hline Generation & $\begin{array}{cc}\text { The } & \text { process } \\
\text { dimension } & \end{array}$ & time & feature \\
\hline The first & $1500 \mathrm{~nm}$ & 1978 & $\begin{array}{c}\text { G line } \\
\text { photoetching machine }\end{array}$ \\
\hline The second & $800 \mathrm{~nm}$ & 1988 & $\begin{array}{c}\mathrm{g} / \mathrm{i} \text { line } \\
\text { photoetching machine }\end{array}$ \\
\hline The third & $500 \mathrm{~nm}$ & 1991 & \\
\hline The fourth & $350 \mathrm{~nm}$ & 1995 & \\
\hline The Fifth & 250nm & 1997 & $\begin{array}{l}\text { KrF } \\
\text { Photoetching } \\
\text { machine }\end{array}$ \\
\hline The sixth & $180 \mathrm{~nm}$ & 1999 & \\
\hline The seventh & 130nm & 2001 & $\begin{array}{l}\text { ArF } \\
\text { Photoetching } \\
\text { machine }\end{array}$ \\
\hline The eighth & $90 \mathrm{~nm}$ & 2005 & \\
\hline The ninth & $65 \mathrm{~nm}$ & 2007 & ArF immersion \\
\hline The tenth & $45 \mathrm{~nm}$ & 2010 & \\
\hline The eleventh & $32 \mathrm{~nm}$ & 2013 & EUV \\
\hline The twelfth & $22 \mathrm{~nm}$ & 2014 & \\
\hline The thirteenth & $14 \mathrm{~nm}$ & $2015 ?$ & \\
\hline
\end{tabular}

$14 \mathrm{~nm}$ process is likely to be a ridge, $193 \mathrm{~nm}$ immersion lithography technology used by the plus two times patterning technology has achieved mass production of the $20 \mathrm{~nm}$ technology, but if not improved source reducing wavelength and uses three times more complex graphics to achieve $14 \mathrm{~nm}$ process exposure technique, not only will greatly increase the exposure times and cost, physical limits may also reach the device and cause failure . Fabrication of feature sizes of $10 \mathrm{~nm}$ has been demonstrated in production environments, but not yet at rates needed for commercialization. However, this is expected by 2016.

At present, most new CPU lithography is the Holland ASML TWINSCAN NXE:3300B. Is currently the highest level of the industry. The system has done a single exposure to $13 \mathrm{~nm}$, and has the ability to reach 9nm,single digit nanometer era laid the foundation for semiconductor process into. ${ }^{[10]}$

\section{References}

[1] Jaeger, Richard C. (2002). Introduction to Microelectronic Fabrication(2nd ed.). Upper Saddle River: Prentice Hall.ISBN0-201-44494-1.

[2] "Semiconductor Lithography (Photolithography) - The Basic Process".

[3] Zhao, X-A; Kolawa, E; Nicolet, M-A (1986). "Reactions of thin metal films with crystalline and amorphous Al2O3".California Institue of Technology.

[4] Nalamasu, Omkaram, et al."An Overview of Resist Processing for DUV Photolithography".

[5] http://Wikipedia.org."AN-Methyl-2-Pyrrolidone".

[6] Madou, Marc (2002).Fundamentals of Microfabrication. Boca Raton, Florida: CRC Press. p.9.ISBN 0-8493-0826-7.

[7] Greener, Jesse; Li, Wei; Ren, Judy; Voicu, Dan; Pakharenko, Viktoriya; Tang, Tian; Kumacheva, Eugenia (2010). "Rapid, cost-efficient fabrication of microfluidic reactors in thermoplastic polymers by combining photolithography and hot embossing".Lab on a Chip10(4): 
522-4.doi:10.1039/b918834g.PMID20126695.

[8] Lithography experts back higher magnification in photomasks to ease challenges// EETimes 2000

[9] Y. Sato et al.,Proc. SPIE, vol. 4889, pp. 50-58 (2002).

[10] http://www.eetimes.com/electronics-news/ASML 